\title{
Metamorfosi del paesaggio nella pianura fluviale mantovana (secoli VIII-XIII)
}

\author{
Metamorphosis of the landscape in the Mantuan river plain \\ (8-13th centuries)
}

GIUSEPPE GARDONI

\begin{abstract}
Tosto che l'acqua a correr mette co, non più Benaco, ma Mencio si chiama

fino a Governol, dove cade in Po. Non molto ha corso, ch'el trova una lama,

ne la qual si distende e la 'mpaluda;

e suol di stare talor esser grama

(Dante Alighieri, Inferno, XX, 76)
\end{abstract}

Author:

Giuseppe Gardoni

Accademia Nazionale Virgiliana (Mantova,

Italy)

ggardoni@tiscali.it

https://orcid.org/0000-0003-1703-0487

Date of reception: 26/04/21

Date of acceptance: 02/08/21

Citation:

Gardoni, G. (2021). Metamorfosi del paesaggio nella pianura fluviale mantovana (secoli VIII-XIII). Anales de la Universidad de Alicante. Historia Medieval, (22), 105-131.

https://doi.org/10.14198/medieval.19812

(C) 2021 Giuseppe Gardoni

Licence: This work is licensed under a Creative Commons Attribution 4.0 International License (CC BY 4.0).

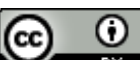

\section{ESTRATTO}

Il presente lavoro delinea le metamorfosi che il paesaggio della campagna ha subito nel cuore della Pianura Padana nei secoli VIII-XIII. Viene presa in esame la documentazione edita e inedita riguardante il territorio mantovano e in modo particolare la zona a ridosso del fiume Po. Per seguire i cambiamenti che l'area oggetto d'esame subì nel periodo considerato ci si soffermerà sulla presenza di aziende agrarie grandi e piccole nell'alto medioevo e i successivi interventi volti a ampliare le superfici coltivate. In quei secoli gli uomini, soprattutto per iniziativa soprattutto della Chiesa mantovana e del monastero di San Benedetto Polirone ( $m a$ anche il comune cittadino fra XII e XIII secolo favorì la messa a coltura di zone boschive), iniziarono una vera e propria lotta all'incolto con opere di disboscamento e di bonifica delle paludi, attività che qualche volta dovettero essere inutili per la forza delle acque: ciò emerge con evidenza soprattutto relativamente alle terre del monastero 
di San Benedetto grazie ad alcune lunghe deposizioni rese nel corso di una controversia. Tuttavia al principio del secolo XIII in vari luoghi le terre coltivate avevano preso il posto dei boschi tanto che risultavano essere "ad usum panis reducte".

PAROLE CHIAVE: secoli VIII-XIII; Pianura Padana; territorio mantovano; boschi; paludi; acque; aziende agrarie.

\section{ABSTRACT}

This essay depicts the transformation that the agricultural landscape underwent in the Po valley between the $8^{\text {th }}$ and $13^{\text {th }}$ centuries. Documents concerning the Mantuan territory, and especially the area next to the Po river, were taken into consideration. Mainly at the behest of the Mantuan Church and of Saint Benedict Polirone's monastery, people started to chop trees down and to reclaim swamps, which often resulted in being useless owing to the water strength. However, at the beginning of the $13^{\text {th }}$ century farmed fields had already replaced plenty of woods, which turned out to be "ad usum panis reducte".

KEYWORDS: $8^{\text {th }}-13^{\text {th }}$ centuries; the Po valley; Mantuan territory; woods; swamps; water; farms.

Agli inizi del Duecento nella pianura mantovana gli interventi di colonizzazione apparivano essere stati tanto massicci che in vari luoghi le terre incolte risultavano essere già ridotte a coltura. Dovette trattarsi di un processo di ampia portata se i vertici del capitolo della cattedrale nel $1223^{1}$ osservarono, con sorpresa, che quelle a loro appartenenti site in Casaletto - località posta sulla sinistra del fiume Mincio - erano "roncate et arate, et de nemoribus et paludibus tracte et ad usum panis reducte" (Torelli, 1930, pp. 153-154). Sembrerebbe d'essere di fronte ad un'eloquente attestazione della definitiva vittoria del coltivato sull'incolto, la cui diffusa presenza si è soliti additare quale caratteristica precipua del paesaggio del primo medioevo. Non solo: è quello un indizio della nuova attenzione e della preoccupazione per i destini del patrimonio boschivo (Cortonesi, 2006, p. 23).

Nelle pagine che seguono, per illustrare le metamorfosi subite dal paesaggio agrario padano dall'alto al pieno medioevo, prenderò in esame a titolo d'esempio il territorio mantovano, ricco di corsi d'acqua piccoli e grandi, e altrettanto ricco di paludi e di boschi (Gardoni, 2010). In quei secoli l'uomo intervenne per sottrarre ampie porzioni di terra alla natura per renderla coltivabile ma non di rado tale faticosa 'lotta' all'incolto - dal quale peraltro si traevano importanti risorse - si rivelò

1 Torelli, 1924, n. LXIV, 1223 ottobre 10.

Abbreviature utilizzate: AG: Archivio Gonzaga; ASMi: Archivio di Stato di Milano; ASMn: Archivio di Stato di Mantova; PF: Pergamene per Fondi. 
effimera. E ciò è vero soprattutto per le terre a ridosso del grande fiume Po. Là la forza delle acque rendeva ancor più arduo, e qualche volta persino inutile, l'intervento dell'uomo, tant'è che nel corso dei secoli considerati e a seconda dei luoghi anche il paesaggio mantovano conobbe varie metamorfosi (Sereni, 1963; Comba, 1983; Montanari, 1984; Menant, 1993; Cortonesi, 2006; Andreolli 2015; Rao, 2015; Rao, 2016). Lo farò non mediante una disamina esaustiva delle fonti disponibili, bensì attraverso qualche significativa esemplificazione, quindi senza avere la pretesa d'essere esaustivi; i limiti di spazio imposti non permettono neppure di considerare la contrattualistica e le fonti normative (Andreolli, 1999; Cortonesi, 2006, pp. 3639; Pasquali, 2008), ad esempio; né d'indugiare in comparazioni con aree finitime a quella qui presa in esame (Castagnetti, 1977, pp. 35-138; Menant, 1993; Varanini, Saggioro, 2008; Saggioro, 2010; Mancassola, 2012, pp. 115-129; Varanini, Saggioro, 2012; Galetti, 2019; Cavalazzi, Mancassola, 2021).

\section{TRA PICCOLI E GRANDI FIUMI}

Solcato dal Mincio e delimitato a sud dall'instabile e mutevole corso del fiume Po (Calzolari, 1998, pp. 4-6; Parmigiani, 2016; Parmigiani, 2019), il Mantovano era segnato ad ovest dall'Oglio e dal Chiese, mentre ad est lo era dal sistema TartaroTione e dall'Allegrezza (Figura 1). A questi corsi va aggiunta la ricca rete di fiumi minori dell'alta pianura alimentati anche dalla fascia dei fontanili: ricordiamo l'Osone, il Birbesi e il Solfero che sfociavano entrambi nel Caldone e quindi nel Mincio, per tacere di tutti gli altri canali e fossati. Il Mincio attorno e oltre la città originava i noti laghi che facevano - e fanno - da corona a Mantova. Appena dopo la città si restringeva a Formigosa per allargarsi poi, ancora con il nome di lago di Mantova, in nuove paludi (Virgiliana, Bugni della Zaita, Bagnolo) che si estendevano fin quasi alla foce in Po (Torelli, 1930, pp. 99-104; Camerlenghi, 2005).

Pure sulla sinistra del Mincio, verso il Veronese, scorrevano acque minori, come l'Essere, e nell'estremo oriente il Tartaro che s'impaludava presso Ostiglia, dopo aver comunicato con il Po mediante una fossa detta Olobia (Libiola).

Nella fascia meridionale la situazione idraulica doveva presentarsi assai più intricata. È necessario al riguardo precisare che, come ho fatto poco sopra, non mi addentrerò nemmeno per quest'area in una dettagliata analisi geomorfologica, aspetto che esula dal presente lavoro, così come non indugerò neppure sul progressivo spostamento dell'alveo del Po o sulle modificazioni dei corsi di altri fiumi rinviando alla letteratura disponibile (Calzolari, 1989, Calzolari, 1998; Marchetti, 2002; Marchetti, 2010; Brandolini, 2018; Brandolini, 2019; Brandolini, 2020; Brandolini, Carrer, 2020). Il Po dall'età tardoantica ebbe vari corsi. Quello che in età altomedievale lambiva Luzzara, Suzzara, Gonzaga e l'odierno San Benedetto Po si disattivò nel corso dell'XI secolo e venne denominato Padus vetus, mentre 


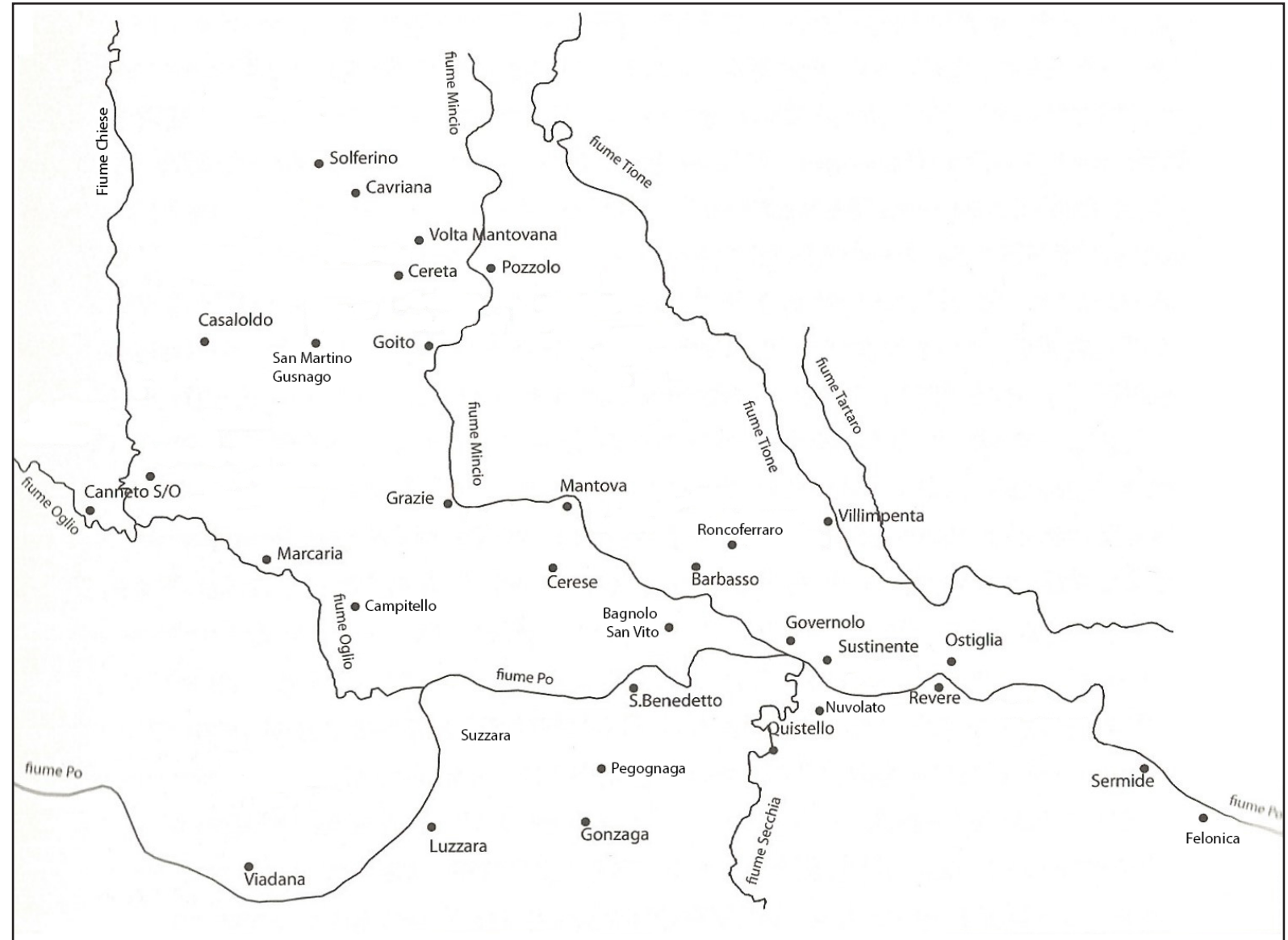

Figura 1. Il territorio mantovano con le principali località menzionate nel testo (disegno dell'arch. Silvia Longo).

un altro ramo, il Larione/Largione/Lirone, finì per diventare il nuovo corso del fiume. Un altro percorso secondario prendeva il nome di Zara e confluiva nel ramo principale del Po vecchio vicino a Pegognaga (Bonacini, 2001; Cavalazzi, 2019). Si devono poi menzionare altri corsi d'acqua che attraversavano le terre poste a ridosso del grande collettore padano: fra i maggiori il Crostolo, il Secchia, il Bondeno (Calzolari, 1998, pp. 7-14). Tra i vari rami del Po si svilupparono vere e proprie isole, spazi insediativi e fondiari (ad esempio le isole di Suzzara, Mauritula, Polirone e Revere). Quella porzione di pianura padana si caratterizzava insomma per la presenza d'un complicato reticolo idrografico, di grande instabilità, causa dell'impaludamento delle aree poste tra dossi fluviali e vaste aree vallive, indicate nei documenti con i termini lacus, palus, vallis.

Erano proprio i principali di tali fiumi, primi fra tutti il Mincio e il Po, a costituire i punti di riferimento utilizzati nella documentazione pubblica per delimitare i confini entro i quali si andò definendo il Mantovano (Colorni, 1959, pp. 53-67). Pur senza entrare in una esaustiva analisi dei diversi esempi adducibili, sarà sufficiente richiamare la descrizione presente nel diploma di Berengario alla Chiesa di Mantova 
dell'anno $894^{2}$, ripetuta in varie conferme successive (Colorni, 1959, p. 53). Merita d'essere ricordato che con la concessione berengariana vengono riconosciuti all'episcopio importanti diritti fiscali: il teloneo, il ripatico, la palifittura sulle rive della città e nel porto, ed anche i diritti sui mercati annuali di tutto il comitato e quelli derivanti dai mulini alimentati dai fiumi (Gardoni, 2006, p. 199). Nel $997^{3}$ Ottone III conferma la Chiesa vescovile nel possesso di tutti i suoi beni e riconosce la detenzione dei diritti di teloneo e di ripatico nella città e nel porto di Mantova, quelli su tutti i mercati annuali che si tengono nell'ambito del comitato, la cui estensione è circoscritta dal Mincio, Larione, Zara, Oglio, Po, Quistello.

\section{PAESAGGI DELLA PIANURA TRA PICCOLE E GRANDI CURTES}

Da tempo la storiografia ha evidenziato, sia pur con diverse sfumature, che la pianura padana doveva in gran parte essere dominata da un paesaggio selvaggio ancora nel secolo XI mettendo peraltro in luce la stretta interdipendenza tra incolto e spazi coltivati già nell'alto medioevo (Fumagalli, 1976, pp. 3-49; Cortonesi, 2006, pp. 1617; Mancassola, 2012, pp. 116-117; Andreolli, 2015, pp. 400-404). Ne è un esempio la corte mantovana di Formigosa, posta fra la città di Mantova ed il Po, che attorno alla metà di quel secolo misurava 3.032 iugeri dei quali ben 3.000 erano ricoperti dal bosco e dalla palude 4 . Pur costituendo un caso limite della bassa padana, esso restituisce un'immagine eloquente della situazione ambientale e del tipo di economia - solitamente definita silvo-pastorale - che vi predominava (Fumagalli, 1986, pp. 57-100; Fumagalli, 1985, p. 31; Fumagalli, 1989, pp. 67-92). Dalle aree incolte gli uomini continuavano a trarre un'infinità di risorse: vi si praticava la caccia e la pesca e si pascolava il bestiame, si raccoglievano i frutti spontanei e si tagliava la legna (Fumagalli, 1992; Andreolli B., Fumagalli V., Montanari M., 1985; Andreolli B., Montanari M., 1988). Tuttavia, come recenti approfondimenti hanno messo in luce, i terreni posti a coltura non costituivano una presenza insignificante nemmeno nel Mantovano anche in età longobarda e carolingia: diversamente da quanto la tradizione storiografica locale asserisce, l'agricoltura non era marginale (Pasquali, 2005, pp. 155-168).

All'età longobarda ci rimanda la conferma di beni al monastero di San Salvatore di Brescia - la dedica a Santa Giulia sarà successiva - effettuata dai re dei Longobardi Adelchi e Desiderio nel $760^{5}$ che riguarda anche 150 iugeri (circa 120 ha) di terre,

2 Schiaparelli, 1903, n. XII, 894 novembre 21: "[...] ambe ripe Mincii de Valegio usque in Largionem fluvium et per Largionem sursum usque Zaram et Olium et deorsum usque Padum per Largionem et per Padum sursum et deorsum usque in Crustellum et Borrigianam".

3 Sickel, 1893, n. 255, 997 ottobre 1.

4 Nicolini, 1949, n. IX, 1072 gennaio 19; osserviamo che vi è una cappella e che fra i confini v'è il fiume "quod dicitur Lario", una fossa "que vocatur Fornicata", il Mincio "quod est lacus".

5 Brühl, 1973, n. 33. 
in parte selvosi, posti nel territorio di San Martino Gusnago e in particolare un casale Secunciolum vicino a Cicognara - nell'odierno comune di Viadana, si suppone, quindi nella bassa mantovana - (Torelli, 1930, p. 119; Pasquali, 2005, pp. 150-160). Doveva trattarsi di un insieme di poderi (si badi, non una curtis) coltivati da 4 massari con le loro famiglie (non è detto se fossero affiancati da servi) i quali avevano a disposizione 300 iugeri di terra (circa 240 ha). È impossibile dire se fosse tutta terra coltivabile e non fossero presenti aree incolte (Pasquali, 2005, p. 160). Il casale situato presso Cicognara - un complesso di beni pari a 300 iugeri - era coltivato da quattro massari con le loro famiglie, ognuna delle quali aveva con ogni probabilità a disposizione dei terreni coltivabili.

Il polittico di Santa Giulia risalente agli anni $879-906^{6}$, registra la presenza sempre in Cicognara di due curtes (Andreolli, Montanari, 1985; Mancassola, 2008; Devroey, Montanari, 2009): una era assai piccola - meno d'un ettaro -, aveva una cappella dotata di sei altari, con ricchi arredi sacri e libri; sull'altra, decisamente più ampia - oltre 400 ha - v'erano 3 case. La pars dominica - quella gestita direttamente - era costituita da quasi 90 ettari di terre coltivate a frumenti, segale, orzo, fava, viti, prato; un mulino ad acqua macinava parecchio grano, rendeva infatti 52 moggi di grano; c'era anche una selva nella quale si potevano ingrassare 200 maiali; ma nell'inventario ne risultano presenti 51 . Vi dovevano vivere un centinaio di persone: 20 schiavi sul dominico, 23 famiglie di coloni sulle 10 sortes del massaricio (terra concessa a massari o coloni). Ogni gruppo familiare doveva corrispondere 3 giornate di lavoro alla settimana. Sul dominico lavoravano numerosi schiavi.

Nell'area più settentrionale del Mantovano erano altresì presenti la corte regia di Pozzolo, sul Mincio (Torelli 1930, p. 117), e almeno una delle diverse curtis possedute da Engelberto di Grimoaldo d'Erbè, così come risulta da un documento dell'846, quella sita "ubi vocabulum est Cereto" - toponimo eloquente, identificabile con approssimazione con l'odierna Cereta, sotto Volta Mantovana, dove è attestata una corte fortificata dal secolo XI (Torelli, 1930, p. 12) -, mentre un'altra era dislocata in Villimpenta (Castagnetti, 1969).

Tra VIII e IX secolo dunque in quella zona l'esistenza di aziende agricole di una qualche consistenza è certa, la loro presenza, le funzioni connesse, dovevano aver in qualche modo intaccato e quindi modificato il paesaggio preesistente; non possiamo però ricostruire in quale maniera la presenza di acque e boschi abbia nel tempo ostacolato quelle attività agricole. Né conosciamo - di queste e pure di quelle di seguito elencate - le vicende successive, sicché, almeno per ora, non si è in grado di dire se la loro attività sia proseguita o quali eventuali modifiche abbiano subito.

Nel polittico del monastero bresciano ci sono altre corti site nel Mantovano: 19 in tutto -5 nell'alta pianura, 14 tra media e bassa pianura -, che costituiscono un

6 Castagnetti et al., 1979, pp. 84-85. 
quarto del totale delle aziende curtensi descritte (70) (Pasquali, 2005, p. 160). Un primo gruppo è dato da piccole corti: Cardulina, Palleriana, Glociano, Cardena, che non sono state identificate, e Cabriana, che si ritiene identificabile con l'odierna Cavriana, in area collinare. Insieme contano un centinaio di ettari di terre coltivate; quella di Cavriana, una delle più grandi, aveva un'ampiezza di 32 ha con oltre 25 individui che vi risiedevano. Non sono menzionati boschi - né altri incolti - ma si conta la presenza di 45 maiali. Ci sono 39 schiavi; sappiamo che a Cavriana sul dominico v'erano 15 servi, 5 maschi, 3 donne, 7 giovani tra maschi e femmine. In quelle cinque corti risalta il ruolo occupato dalla coltivazione della vigna: a Palleriana su 4 ettari di terra coltivata c'è un vigneto di mezzo ettaro. C'era un'altra coltura specialistica, quella dell'olivo. Nei magazzini erano presenti miglio, meliga e panico, che si può ritenere fossero proprio il prodotto della lavorazione di quelle terre. Vengono elencati i coloni (20 capofamiglia) insediati sul massaricio, una piccola società con una sua propria gerarchia. Insomma, quelle descritte sono terre particolarmente vocate a colture specializzate per la loro ubicazione e erano di certo da tempo intensamente coltivate.

La stessa fonte ci informa della presenza di un altro gruppo di corti mantovane, sempre sulla destra del Mincio: Gummolf, Gutus, Marcharegia, Capadello, Cubolas, Riveriolas, Mativado. Di queste, 5 hanno una cappella, ognuna delle quali ha propri libri e arredi sacri; 6 sono dotate di un mulino ad acqua. Le terre del dominico sono complessivamente pari a quasi 600 ettari (Pasquali, 2005, pp. 164-165); erano abitate da 89 prebendarii. Quelle massarice invece si estendevano per circa 350 ha e le coltivavano 62 famiglie di manenti e 4 famiglie di liberi; tra il personale 'specializzato' si elenca un porcaio. In questo secondo gruppo di corti c'erano cospicue selve: vi si potevano allevare 1271 maiali - la più grande è quella di Riveriolas, nella quale era possibile ingrassare fino a 560 porci -, ma nell'elenco se ne enumerano 382. Bassa è la presenza della vite. Da quanto detto è possibile dunque sottolineare come anche la bassa pianura nel secolo VIII e soprattutto nel IX fosse interessata da uno sviluppo agricolo di una certa importanza sia pur condizionato dalla presenza delle acque e dove meno importante era, ovviamente, la coltivazione della vite. Nel comitato mantovano Santa Giulia aveva beni siti anche nel luogo denominato Lauriolo vel Canedolo - potrebbe trattarsi dell'odierno Canneto sull'Oglio - dove l'insieme delle proprietà - case, sedimi, viti, prati, boschi e paludi - avevano un'estensione pari a 247 iugeri $^{7}$.

Un altro inventario ci dice qualcosa anche del territorio sinistra Mincio e di altre corti, questa volta soggette al monastero di San Colombano di Bobbio ${ }^{8}$. Sono attestate tre piccole corti: Portu de Mantua, Adarbassio, Fraxeneta, che si reputa fossero dislocate nei pressi delle attuali località di Porto Mantovano, Barbasso e Frassinara. Si tratta di aziende di estensione modesta: due hanno solo il dominico (pochi ettari a

7 Archetti, 2019, n. 8, 980 [maggio 23 o giugno 22].

8 Castagnetti et al., 1979, pp. 121-144: 138-139, 145-165:159-160. 
Porto e dieci circa Barbasso); quella di Frassinara invece ha i caratteri di una classica curtis: dominico e massaricio sono quasi equivalenti, vi abitano 4 famiglie di livellari che non devono alcuna prestazione d'opera, prevalgono i seminativi ma non sono assenti la vite e i prati. Nella parte dominicale della corte di Adarbassio ogni anno si potevano seminare 30 moggia di grano e si producevano 30 anfore di vino, 12 carri di fieno; in quella di Fraxeneta si seminavano 20 moggia di grano, si producevano 20 anfore di vino e 20 carri di fieno. Non c'è cenno alla presenza di aree boschive, né si menzionano maiali. Anche tali aziende, al pari di quelle emerse dall'inventario bresciano, al pari di quelle site nella media pianura, non costituiscono delle aziende di tipo pioneristico (Pasquali, 2005, p. 166).

Nemmeno la media e bassa pianura mantovana, dunque, nei primi secoli del medioevo era del tutto priva di aree intensamente coltivate, in molti casi erano piccole aziende, ma di certo non v'erano solo ampie distese di boschi. Non pare inutile tener conto del fatto che per quell'area sono emerse testimonianze eloquenti della centuriazione romana (Buonopane, 2003, pp. 111-124; Calzolari, 2003, pp. 139-161; Tamassia, 2003, pp. 163-182): in sinistra Mincio, tra il Mincio e il Tione, su una superficie di circa $40 \mathrm{kmq}$ a nord della città, e nelle valli di Roncoferraro su un'area di oltre 70 kmq (Calzolari, 1989, pp. 111-140; Calzolari, 2003, p. 141); nella zona della pianura tra Oglio, Chiese, Po e Mincio se ne riconoscono tracce che giungono contro l'odierno corso del Po; a nord, analoghi interventi dovettero interessare i margini delle colline gardesane. Nel complesso si stima che l'area complessiva abbia interessato circa 450-500 kmq e comprendesse terreni tanto dell'alta quanto della media pianura (Calzolari, 2003, p. 144). Tracce numerose di ville rustiche sono emerse nella zona di Sermide, deducendone che l'area doveva essere densamente abitata, il che non esclude che vi fossero, assieme ad aree coltivate a cereali e a prato, zone boschive, ovvero i saliceti ricordati da Plinio sul corso del Po; una villa con necropoli è emersa a Felonica; manufatti d'età romana sono stati messi in luce a Bagnolo San Vito e a Cicognara (Tamassia, 2003, pp. 167-172). Per l'area dell'Oltrepò spicca la località di Pegognaga (Tamassia, 1996); è poi nota una necropoli nei pressi di San Benedetto Po e qualche reperto è stato rinvenuto pure a Nuvolato (Tamassia, 2003, p. 169). L'elenco potrebbe continuare e potremmo annoverare San Martino Gusnago e ancora più a Nord, il territorio di Cavriana (Tamassia, 2003, pp. 175-176) che parrebbe essere stato molto abitato.

\section{NELLA BASSA PIANURA TRA ACQUE, ISOLE E BOSCHI}

La fascia di pianura costeggiante il Po, per alcuni chilometri a partire dalle rive del fiume, per la sua natura paludosa e per il ricorrente pericolo di piene, non era di certo facilmente colonizzabile, sicché boschi e paludi ne costituiranno a lungo il tratto distintivo (Figg. 2-6) e a lungo quelli saranno ambienti destinati in prevalenza alla 


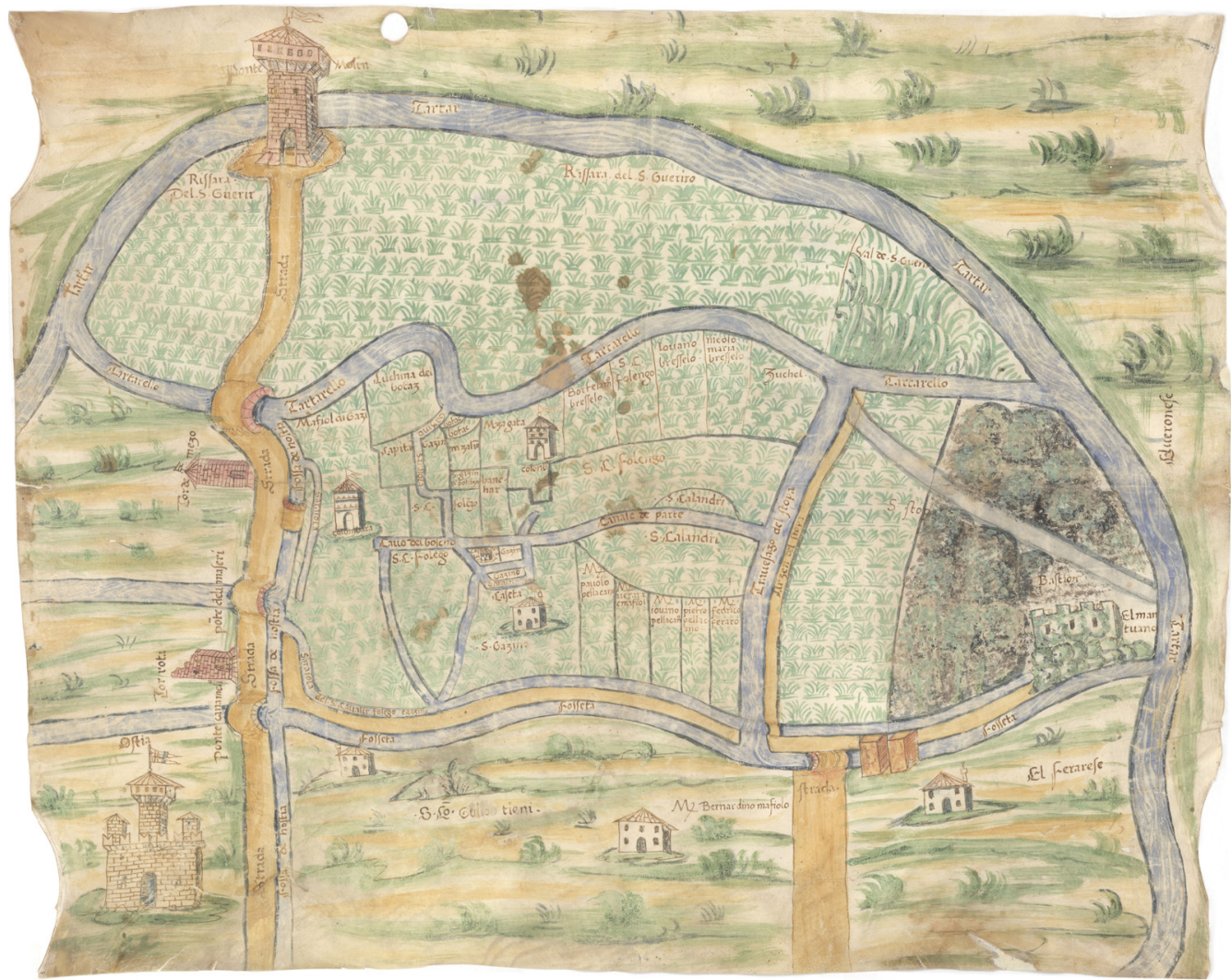

Figura 2. Il territorio di Ostiglia in una mappa su pergamena di fine XV-inizi XVI secolo (ASMn, AG, b. 90 , c. 49r).

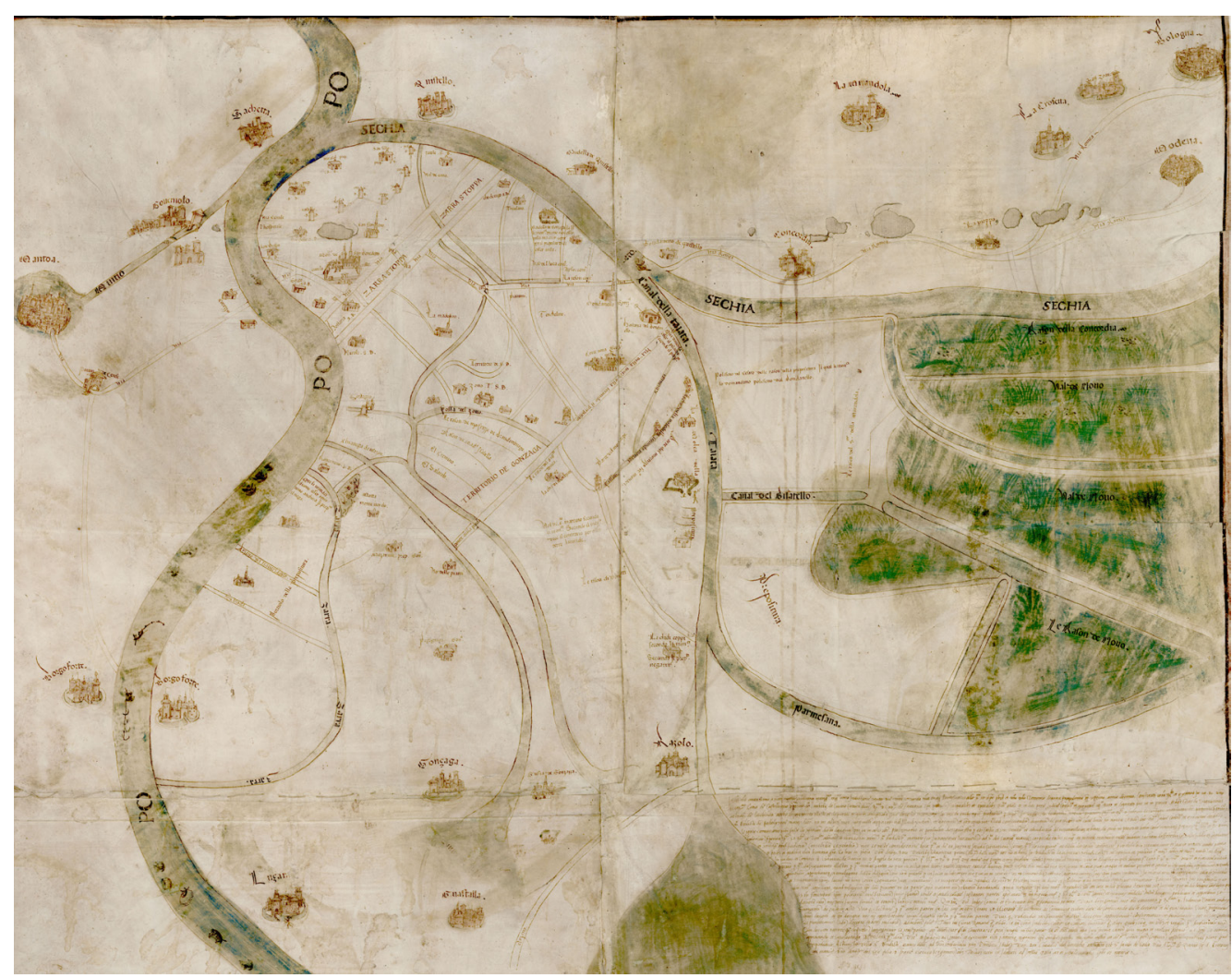

Figura 3. Il territorio di San Benedetto Polirone in una mappa del 1533 (ASMn, AG, b. 90, c. 35r). 


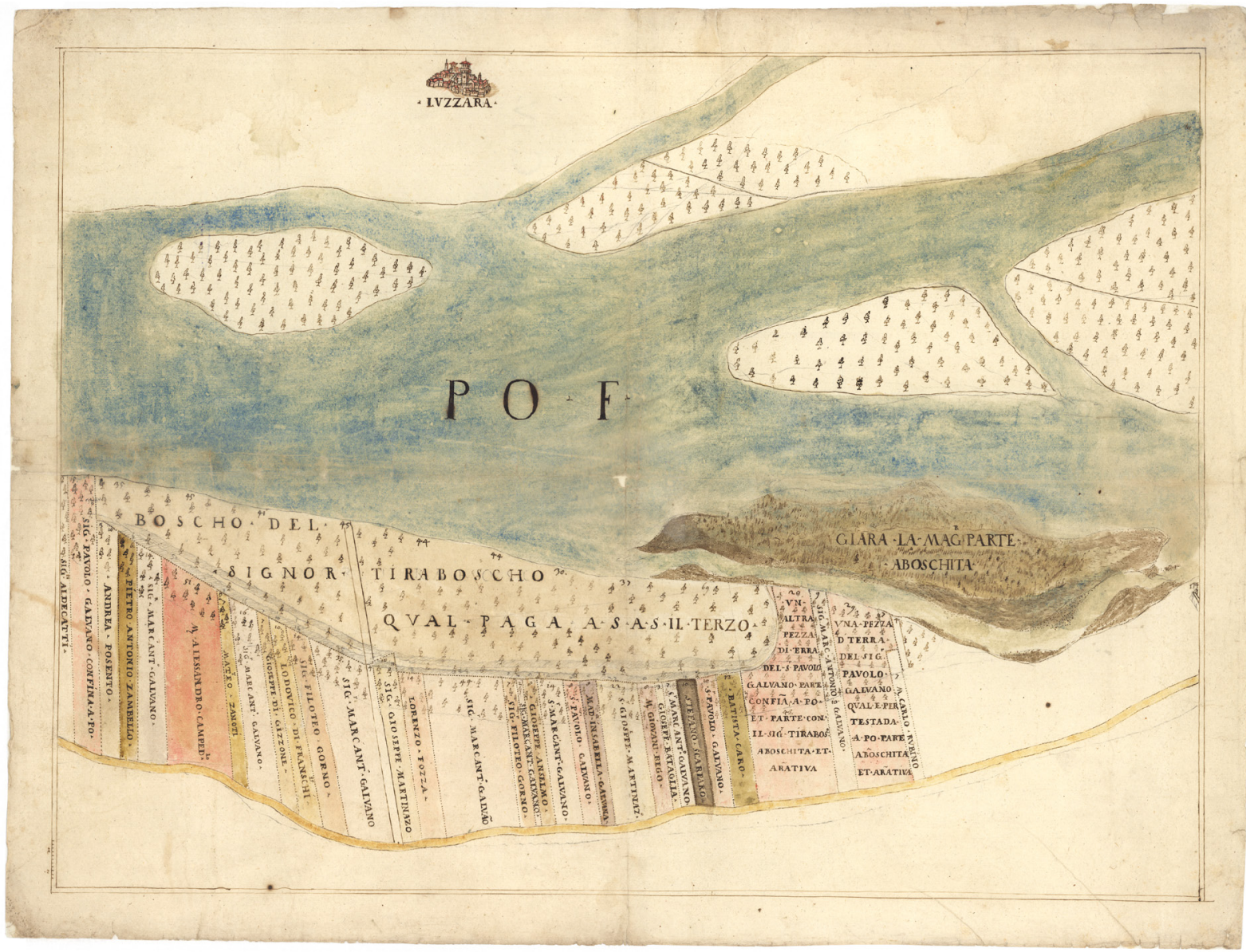

Figura 4. La golena di Dosolo, mappa di metà XVII secolo (ASMn, AG, b. 90, c. 18r).

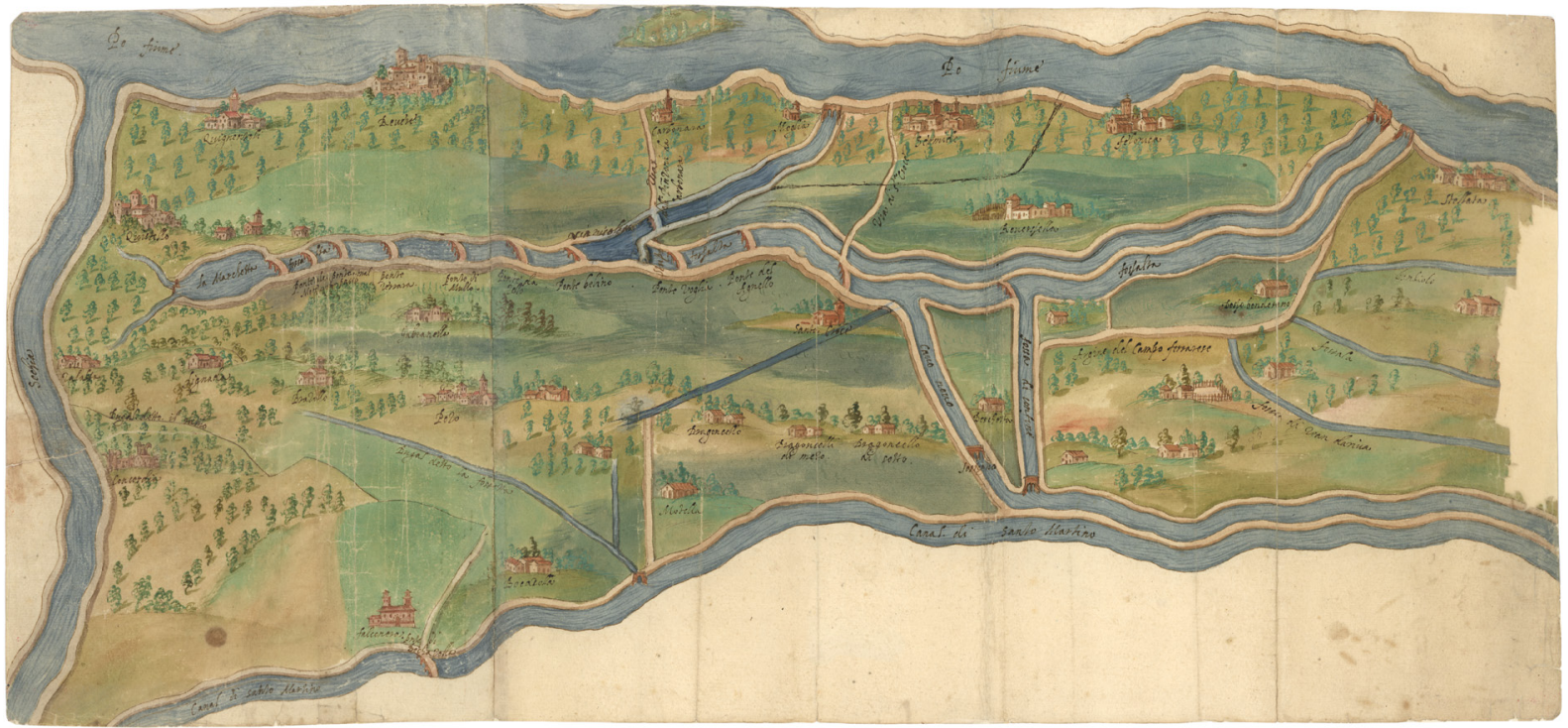

Figura 5. Il territorio dell'Oltrepò mantovano, mappa del secolo XVII (ASMn, AG, b. 91, c. 36r). 


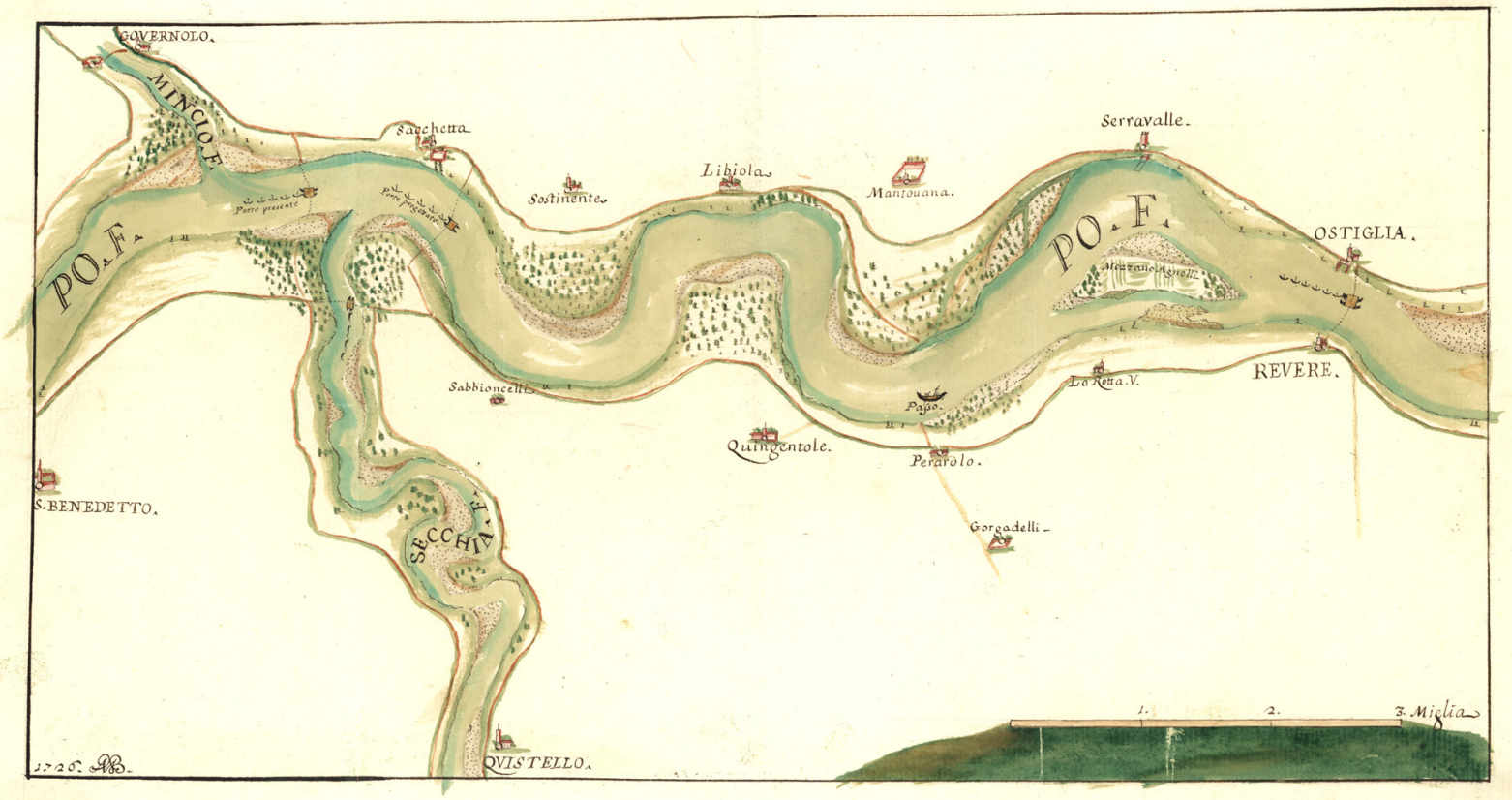

Figura 6. Il corso del fiume Po dalla foce del Mincio sino a Ostiglia in una mappa del 1726 (ASMn, AG, b. 3193, c. 534r).

pesca e alla caccia. Quella parte della pianura mantovana per i secoli anteriori alla dominazione canossiana è scarsamente documentata. Diversa è invece la situazione per le aree contermini alle quali possiamo guardare come a dei termini di paragone (Rinaldi, 2005b, pp. 39-50; Galetti, 2019).

I monasteri traevano indubbi vantaggi e profitti dal farsi concedere ampie estensioni di quelle terre che a noi appaiono selvagge ma dove potevano far pascolare le loro mandrie di animali e catturare pesci e selvaggina, attrarre persone e favorire il sorgere di nuovi stanziamenti o dare vigore a quelli preesistenti. Nell'Oltrepò, tra Sermide e Bondeno, ad esempio, l'abate di Nonantola aveva il diritto sulla metà delle

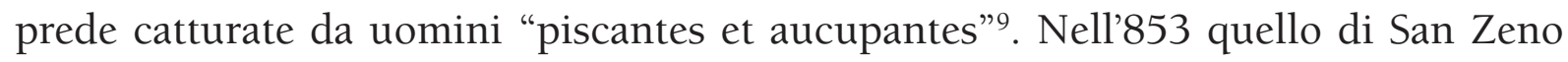
di Verona ottenne la conferma della concessione di poter pascolare "in regalibus tam silvis quam in ceteris pascuis ... greges ovium ... nec non et pecorum imentorumque vel ceterorum animalium" (Fumagalli, 1967, p. 142). Nella prima metà del secolo IX si fece concedere la porzione della foresta ostigliese che non era toccata a Nonantola (Fumagalli, 1976).

Larea più difficilmente colonizzabile doveva essere, come detto, proprio quella prospiciente il Po. Disboscamenti prima del secolo $\mathrm{X}$ sono testimoniati per varie località del Modenese: il presule di Modena nei suoi contratti con liberi coltivatori pattuì l'obbligo di scavare fossati per lo scolo delle acque, costruire l'abitazione e gli edifici agricoli, adattare il terreno alla coltivazione della vigna. Solo in una occasio-

9 Manaresi, 1955, n. 30 (a. 818). 
ne si ordina di procedere al disboscamento ("runcare") (Fumagalli, 1967, p. 143; Castagnetti, Ciaralli, 2011; Mancassola, 2018, p. 89-90). E non si può omettere di fare riferimento ai noti contratti di livello stipulati a Ostiglia per il dissodamento di quella foresta fra l'abate di Nonantola e i liberi livellari ai quali numerose volte ha guardato Vito Fumagalli (Fumagalli, 1967, p. 143;) che tante pagine ha dedicato al nostro argomento (Andreolli, Galetti, Lazzari, Montanari, 2010) parlando proprio di terre e uomini della pianura, impiegando tanto gli esempi già addotti quanto molti di quelli che seguiranno (Fumagalli, 1976). Labbazia di Nonantola negli anni Venti del IX secolo concesse a livello terre a Ostiglia in parte già ridotte a coltura ("ad lavorando") e altre da sottrarre all'incolto ("ad roncando"). E lì vi si producevano grano grosso e minuto, legumi e lino, si prevedeva anche l'impianto della vite (Torelli, 1930, p. 119-120). In anni non lontani da quelli, il medesimo ente concedeva terre presso la "fossa Lubiola" con l'obbligo di realizzarvi casa, corte e orto e di "claudere vineam, pastenare, propaginare, silva infructuosa roncare" (Torelli, 1930, p. 120). Qui dunque da tempo il potente monastero doveva aver avviato un'azione di dissodamento con continuità e con risultati duraturi: anche per gli anni successivi si conoscono infatti contratti di livello aventi per oggetto terre aratorie, orti e vigneti (Torelli, 1930, p. 120).

Sempre al monastero di Nonantola re Astolfo concesse nel 753 metà di Sermide, una proprietà regia dalla quale si ricavava pesce (Torelli, 1930, p. 116). Al principio del secolo successivo da quelle terre si traevano sempre pesce e porci e vi si praticava la caccia (Torelli, 1930, p. 116). Dal già citato elenco dei beni di Santa Giulia di Brescia si apprende che l'ente possedeva un complesso di beni dominici con una peschiera e una selva in Sarmida - identificabile proprio con Sermide -, beni amministrati da un funzionario che vi risiedeva. La curtis di Sermide torna ad essere elencata, assieme a quella di Migliarina (Andreolli, 2000, pp. 423-426) - altra grande proprietà del monastero giuliano - nel documento di Bonifacio di Canossa del marzo $1052^{10}$. E lì il monastero bresciano continuerà a detenere beni nell'XI secolo e in quello successivo (Mancassola, 2019, p. 29).

E sempre nell'inventario dei possessi di Santa Giulia di Brescia si menziona anche Gonzaga, che, citata in documenti successivi, era dotata di 420 iugeri di selva (Torelli, 1930, p. 124 e 137) ${ }^{11}$. Nel 967 la corte di Gonzaga - già incastellata da almeno un anno - i cui confini sono segnati dai fiumi Bondeno, Gonzaga, Po - contava 320 iugeri di selva, quattro tra "sedimina" e viti, 76 di arativo (Torelli, 1930, p. 124). Il fundus di Nuvolato nel 962 era invece costituito da quattro iugeri di terra arativa e 96 di selva (Torelli, 1930, p. 124).

Il bosco dunque doveva essere il tratto distintivo di quel paesaggio (Andreolli, Montanari, 1988), ma la presenza di terre coltivate è segno di un intervento umano

10 Archetti, 2019, n. 15, 1052 marzo 29.

11 Torelli, 1914, n. 34, 967 aprile 22. 
già avviato per non dire consolidato. Infatti già nel corso del secolo IX lungo il Po l'attività di disboscamento in varie località aveva assunto una qualche importanza. Più volte Vito Fumagalli ha addotto ad esempio un falso diploma carolingio datato 781 con il quale si concedevano alla Chiesa di Reggio vari beni tra i quali un bosco regio posto nell'isola di Luzzara - una delle tante isole formate dal Po e dai suoi affluenti - "quod nunc noviter excolitur" (Fumagalli, 1967, p. 141). Su un'altra isola, quella di Suzzara, alla fine del IX secolo c'era una curtis (Torelli, 1930, pp. 122-123): da bene regio divenne proprietà del vescovo di Reggio; vi si trovavano molini, peschiere, paludi e boschi, prati e mansi compresi tra i corsi del Po e del fiume Zara. Su quell'insula doveva trovarsi Torricella, dove tra IX e X secolo erano presenti piccoli poderi (Torelli, 1930, p. 123). Tra le acque del fiume, che non di rado le doveva sommergere, erano site le terre presso Guastalla che alcuni livellari, così come i loro contratti contemplavano, avrebbero potuto abbandonare se fossero state allagate dal Po senza incorrere in alcuna ammenda (Fumagalli, 1967, p. 141; Andreolli, 2000, p. 415). Il pericolo dell'acqua era sempre incombente e poteva in un breve lasso di tempo rendere vano il decennale lavoro dei contadini e quindi mutare il paesaggio e la sua utilizzazione.

\section{PAESAGGI DINAMICI}

Come è stato detto, anche il tratto di pianura delimitato a sud dal Po, ad occidente dal Mincio e ad oriente dal Tartaro, era solcato da numerosi corsi d'acqua, alcuni dei quali anche assai modesti (Calzolari, 1989). Di un tale intricato sistema idrico oggi non permangono che poche tracce: molti dei corsi d'acqua minori sono scomparsi; altri presentano una portata molto ridotta. Dovettero, tuttavia, essere proprio quei corsi d'acqua a costituire la principale via di penetrazione dell'uomo in quelle terre in gran parte ricoperte da boschi caratterizzati dalla presenza di essenze arboree come l'olmo, il carpine, e da numerose valli acquitrinose, laghi più o meno estesi e paludi, una situazione idrografica che ha lasciato testimonianze anche nell'odierna toponomastica. Tra Mantovano e Veronese doveva estendersi un bosco enorme che si dilatava nella pianura veronese sino alla zona paludosa di Ostiglia e Sustinente. Tale foresta dovette essere interessata da azioni di disboscamento e bonifica nel secolo IX che proseguirono sino a quando nella seconda metà del secolo XII ne restava oramai ben poca cosa (Mor, 1964, pp. 57-60; Castagnetti, 1972, p. 32).

Gran parte di questi beni boschivi di proprietà pubblica passò, grazie a donazioni di re e imperatori, ai maggiori enti ecclesiastici dell'Italia padana. Infatti vi possedevano ampiamente - lo si è già rilevato - il monastero di San Silvestro di Nonantola, quello di San Zeno di Verona (Castagnetti, 1977, p. 45) e di San Benedetto Polirone, oltre a chiese vescovili e numerosi altri enti ecclesiastici. Fra i possessi concessi al monastero di San Zeno nel centro demico di Villimpenta - allora in ter- 
ritorio veronese - da Berengario I nell'anno 905"12, compare una "silva que vocatur Carpeneda", confinante con Nosedole - oggi frazione di Roncoferraro -, la fossa Pomanon, l'Agricia e Corvolata (Castagnetti, 1974-1975, pp. 85-88; Calzolari, 1989, pp. 51-54). Non è possibile appurare con certezza se questa Carpeneda sia la stessa Carpeneta che sappiamo essere appartenuta fra i secoli XI e XII ai cittadini-arimanni di Mantova (Castagnetti, 1996, pp. 117-147; Gardoni, 2007): è più realistico pensare a diversi lembi di quella vasta area boschiva estendentesi a cavallo fra i territori di Verona e Mantova cui si è accennato sopra, separati dall'Agricia maior, il cavo Allegrezza (Castagnetti, 1974-1975, p. 88). Tale ipotesi sembra trovare conferma nella documentazione mantovana del secolo XII, che permette di constatare l'ubicazione di Carpeneta, fitotoponimo che tradisce la presenza di carpini (Calzolari, 1989, p. 66). Nel 1125 vengono citati, uno dopo l'altro, i boschi di Vallarsa, Poletto "et Carpineta"13. Qualche decennio più tardi ${ }^{14}$, fra le coerenze di appezzamenti posti nel territorio di Carzedole - identificabile con l'odierna Villa Garibaldi in comune di Roncoferraro - si citano il "nemus Carpenete" ed il "nemus comunis", parti di una stessa area boschiva (Torelli, 1930, p. 36). In un documento del $1176^{15}$ i confini della corte di Barbasso sono dati dal corso d'acqua Derbasco (Paglia, 1879, p. 268), dal "tilionem que apellabatur Agritia" - il cavo Allegrezza, già menzionato -, "tillionus" che "claudit Carpenetam totam et Cuguzum et Silvorum et Valarsam et Nivilariam et Politum usque in Diruptam" (Castagnetti, 1974-1975, pp. 84-85 e 8889). La selva Carpeneta insisteva quindi all'interno di una ben più vasta area posta sulla sinistra del fiume Mincio, posta ad ovest del Tione, a nord di Nosedole e di Vallarsa - località quest'ultima non molto discosta da quella denominata Poletto -, e a est dei territori di Carzedole e Barbasso.

Entrambi i lembi boschivi nel corso del secolo XIII vennero sottoposti ad un'intensa azione colonizzatrice: quello veronese, ad oriente dell'Allegrezza, fu preso d'assalto sotto la guida di enti ecclesiastici - San Zeno e Santa Maria in Organo - e spezzettato in unità minori (Calzolari, 1989, p. 57); quello mantovano, ad occidente dell'Allegrezza, risulta essere, in parte, anche nella disponibilità del comune cittadino che, seguendo forse un sistema di ripartizione in mansi messo in atto in altre località del territorio soggetto, fra le quali va annoverata Poletto, lo assegnò a privati promuovendone la messa a coltura (Torelli, 1930, pp. 149-154). Nel 120216, ad esempio, il comune urbano cedette a dei privati due mansi di terra con bosco "in nemore Carpenete, loco et fundo qui dicitur Coazia". Immobili del comune in questa medesima località continueranno ad essere menzionati nella documentazione

12 Schiaparelli, 1903, n. LXII.

13 Rinaldi et al., 1993, n. 113, 1125 dicembre 1.

14 Torelli, 1914, n. 325, 1164 aprile 6.

15 Torelli, 1924, n. XXIX, 1176 giugno 11.

16 ASMn, AG, b. 302, n. 415, 1202 settembre 23. 
degli anni successivi ${ }^{17}$. Le attestazioni posteriori sino ad ora raccolte permettono di constatare $^{18}$, oltre alla persistenza del toponimo, l'esistenza di aree incolte, la prosecuzione dell'attività di messa a coltura di nuove terre destinate a coltivazioni cerealicole nonché all'impianto della vite (Pini, 1989; Gaulin, Grieco, 1994; Archetti, 1998).

Quelle terre furono al centro di più contese tra XII e XIII secolo. Un documento del $1125^{19}$ riferisce infatti di una lite avente per oggetto i confini di terre site in Vallarsa, Poletto e Carpeneta e lo sfruttamento delle stesse da parte degli abitanti di Casale, Sustinente e Libiola; sennonché tale documento è stato recentemente ritenuto un falso la cui 'fabbricazione' risalirebbe ad un secolo dopo, quando, come vedremo oltre, appaiono testimonianze certe dell'esistenza di una vertenza fra i due enti concernente quegli stessi beni.

Sul finire del secolo evidenti appaiono i tentativi del comune di Mantova di superare i confini del suo contado verso il territorio di Verona, e viceversa. Nella pace stipulata fra le due città nel 1202, dopo il divieto di erigere castra e munimenta sino a quando la quaestio "de episcopatu vel districtu" non sarà definita - termini indicanti i territori afferenti ai centri urbani - e dopo la promessa di osservare un arbitrato per la "discordia campanee" (Castagnetti, 1990, pp. 153-160), si assicura di mantenere fede agli accordi che saranno raggiunti in merito alla "discordia Poleti" ${ }^{20}$. Nei decenni seguenti i contrasti non vennero meno. Il documento seguente mostra in particolare come il comune mantovano tutelando il monastero di San Benedetto tutelasse i propri interessi da minacce esterne. Sul finire del $1228^{21}$ sono in atto tentativi per la composizione del contenzioso "super facto concordie de Poleto et Busca inter abbas Sancti Benedicti et Sancti Zenonis". Gli incaricati della composizione della vertenza da parte del legato pontificio - l'arciprete mantovano e futuro vescovo Pellizzario ed un canonico bresciano - esposero alle autorità pubbliche della città di Mantova i termini dell'accordo: il territorio di Poletto avrebbe dovuto essere diviso in due parti uguali da assegnare a San Benedetto e al monastero veronese di San Zeno. Il podestà ed il consiglio espressero parere favorevole in merito alla ratifica della concordia nei termini esposti qualora "conditiones pro nostro comuni Mantue et nostro abbate Sancti Benedicti meliorari non possunt". La vicenda vedeva coincidere gli interessi

17 Tra i vari esempi adducibili si vedano ASMn, AG, b. 302, n. 435, 1202 ottobre 17; ASMn, AG, b. 302, n. 440, 1202 novembre 19; ASMn, AG, b. 302, n. 461, 1203 gennaio 31.

18 ASMn, AG, b. 303, 1225 gennaio 31; ASMn, AG, b, 303, 1225 giugno 30; ASMn, AG, b. 303 bis, 1228 febbraio 11; ASMn, AG, b. 303 bis, 1228 maggio 24; ASMn, AG, b. 303 bis, 1228 ottobre 30; ASMn, AG, b. 303 bis, 1228 febbraio 4; ASMn, AG, b. 303 bis, 1229 settembre 16; ASMn, AG, b. 303 bis, 1229 ottobre 30; ASMn, AG, b. 303 bis, 1235 maggio 2; ASMn, AG, b. 303 bis, 1239 novembre 28; ASMn, AG, b. 304 bis, 1250 maggio 11; ASMn, AG, b. 238, 1252 marzo 13; ASMn, AG, b. 304 bis, 1260 gennaio 12.

19 Rinaldi et al., 1993, n. 113, 1125 dicembre 10.

20 Navarrini, 1988, nn. 31-32, [1202].

21 ASMi, PF, b. 208, 1228 novembre 9. 
di San Benedetto con quelli del comune cittadino, entrambi interessati a controllare aree poste sullimportante confine con il Veronese, anche se per motivi diversi ancorché complementari. Al comune premeva il controllo di un'area di confine di notevole importanza politica e militare, importanza sottolineata dal divieto di erigere "munitionem" o "hedifitium ad guerram" che, tanto al comune mantovano quanto a quello veronese, venne richiesto di rispettare. Venti giorni più tardi i monasteri di San Zeno e di San Benedetto provvidero a dividere i loro beni presso Poletto ${ }^{22}$. Nel relativo documento si dispose che entrambi gli enti potessero erigere una villa sulla parte di proprietà loro spettante, ma per evitare che l'una potesse minacciare l'altra si vietò, fra le altre cose, di edificare "castrum", "motta", casatorre o altri edifici fruibili per scopi di difesa e di offesa; ogni nuovo centro demico avrebbe potuto essere cinto da un fossato e circondato solo da una siepe e da due porte lignee (Castagnetti, 1977, 81-82).

Il territorio di Poletto continuò ad essere al centro di contrastanti interessi anche negli anni successivi. Il comune di Mantova tentò di ottenerne il controllo a detrimento del cenobio polironiano, come attesta l'intervento a difesa delle prerogative di San Benedetto, su incarico del legato pontificio, del frate predicatore Guala nell'anno $1229^{23}$. Nonostante tale intervento, il comune di Mantova non desistette dai propri intenti. Nel $1235^{24}$ il podestà Giacomo "de Mellate", togliere la virgola incaricò tre uomini affinché indagassero "super comunibus civitatis Mantue" e specificatamente "super determinatione, dessignatione, divisione et confinatione in Polleto facienda". Essi procedettero all'escussione di numerosi testimoni e all'esame di numerose prove documentarie, fra le quali viene annoverato il citato atto divisionale del 1228 con San Zeno, giungendo al riconoscimento dei diritti che San Benedetto vantava su Poletto, compreso quello di "claudere et cavare sine molestatione comunis Mantue" 25 (Castagnetti, 1974-1975, pp. 103-104).

Un esempio ulteriore di un insediamento sviluppatosi in prossimità dell'alveo padano è costituito da Sermide, dove, come detto, vantava diritti sin dal secolo IX il monastero di San Silvestro di Nonantola ed anche quello bresciano di Santa Giulia (Castagnetti, 1982, 78-79; Castagnetti, 2006, p. 33). La corte fu assegnata con il relativo castello all'episcopio mantovano nel secolo X, come appare dal privilegio di Ottone III del $997^{26}$ (Gardoni, 2006, p. 215). La pieve locale è contemplata fra quelle soggette alla Chiesa di Mantova ${ }^{27}$. Stando ad un noto documento del $1082^{28}-$ documento di dubbia autenticità invero (Gardoni, 2007, pp. 329-334) - il vescovo man-

22 ASMn, AG, b. 10, n. 39, 1228 novembre 28.

23 ASMi, PF, b. 208, 1229 giugno 14.

24 ASMi, PF, b. 208, 1235 settembre 13.

25 ASMi, PF, b. 208, 1235 settembre 18.

26 Sickel, 1893, n. 225, 997 ottobre 1.

27 Bresslau, 1909, n. 235, 1037 marzo 31.

28 Torelli, 1914, n. 101, [1082] maggio 5; Goez, Goez, 1998, pp. 470-474, + A3. 
tovano Ubaldo, su sollecitazione della contessa Matilde, investì il nipote Ubaldo, a nome dei figli del fratello, di una vera e propria signoria su Sermide, concedendo ad essi la curtis e ampi diritti giurisdizionali con esclusione dei beni dell'abbazia di San Maria di Felonica, della chiesa locale e dei diritti di pesca dei monasteri di Santa Giulia di Brescia e di San Ruffino di Mantova. Ai nuovi domini spetterà ricevere la terza parte del pesce pescato in tutto il territorio concesso, le decime di tutti i frutti della terra, delle viti, dei maiali e degli animali da cortile, delle uova e "duos sturiones magnos de Pado" (Fumagalli, 1976, pp. 10-11). Nel 1205 metà della curia di Sermide risulta soggetta al monastero bresciano di Santa Giulia che l'aliena in quell'anno assieme ai diritti giurisdizionali ${ }^{29}$ : nel documento viene fatto riferimento ad otto piscatores, soggetti al monastero che hanno diritto di pesca. Questi otto dipendenti del monastero bresciano, abitanti in Sermide e dediti appunto alla pesca, erano già eccettuati nell'investitura vescovile sopra citata del 1082. Importa richiamare anche una convenzione stretta fra i Visdomini e gli abitanti di Sermide nell'anno $1207^{30}$ nell'ambito della quale si regolamenta l'uso delle acque e dei canali comuni, un uso che viene riconosciuto alla comunità rurale dietro la corresponsione di un terzo dei pesci, e dove si fa riferimento anche alla possibilità che il Po inondi e alle consuetudini di pesca proprie di quegli uomini (Torelli, 1930, p. 196, nota 1).

\section{PAESAGGI INSTABILI A RIDOSSO DEL PO TRA X E XII SECOLO: NUOVI EQUILIBRI}

In quelle terre della bassa padana, "nella solitudine di un paesaggio ancora in gran parte deserto" (Fumagalli, 1976, p. 9), Tedaldo di Canossa fondò, nell'isola fluviale omonima, circondata dalle acque del Po e del Lirone, il monastero di San Benedetto (Rinaldi 1998; Bonacini, 2000; Golinelli, 2008). Il fondatore cedette alla neonata comunità numerosi beni e un piccolo gruppo di servi ${ }^{31}$. Ad essi doveva spettare la cura dei campi, delle valli, dei boschi, dei canali come a quella coppia di coniugi, con figlio, che assieme ad altri quattro uomini erano deputati allo sfruttamento forestale e vallivo. Negli anni successivi con numerosissime donazioni il patrimonio monastico si ampliò notevolmente arricchendosi, tra l'altro, di ampie porzioni di suolo limitrofo al Po, donazioni che comprendevano tanto aree incolte quanto terre coltivate da tempo. Non è mia intenzione soffermarmi su di esse e nemmeno addentrarmi in una esaustiva ricostruzione del patrimonio terriero dell'ente, basterà anche in questo caso qualche esempio (Andreolli, 1998, 141-151). Nel 1110 in quel luogo risulta sorgere già un centro fortificato e vi sono attestati 1000 iugeri di terra (Torelli, 1930, p. 122). Nel 1074 erano stati donati al monastero campi e vigneti in località

29 Franceschini, 1991, n. 46, 1205 dicembre 1.

30 ASMn, AG, b. 3385, 1207 maggio 13 (copia autentica del secolo XIII).

31 Rinaldi et al., 1993, n. 14, 1007 giugno. 
prossime a Roncoferraro: terre per complessivi 3 iugeri lavorate da due fratelli ${ }^{32}$. Nel 1096 Matilde dovette invece intervenire per risolvere una vecchia questione centrata su terreni sull'isola di San Benedetto di fresca messa a coltura circondati da ogni parte dall'acqua e dalla palude ${ }^{33}$. Ebbene, in un paesaggio dominato da fossi, canali navigabili, e macchie selvose - un paesaggio destinato a rimanere tale a lungo (Figura 3) -, vi si coltivavano cereali. Nel 1113 Matilde donerà un manso in insula Reveris con bosco e pascolo ${ }^{34}$. Con un'altra delle tante donazioni matildiche ai monaci sono concessi diritti legati allo sfruttamento dei boschi e in modo particolare per l'allevamento allo stato brado dei porci con limitazioni per la cattura della selvaggina ${ }^{35}$ : in questo documento si legge che "homines monasterii ad aidam que ad bestias capiendas in forestis fieri solebat ulterius non conpellantur, aut etiam vocentur ut eam faciant" (Torelli, 1930, p. 118). Ecco dunque una bella immagine degli uomini che sulle terre monastiche esercitavano la caccia. L'anno prima ${ }^{36}$, Matilde di Canossa autorizzò l'uso d'un bosco nella pianura mantovana prescrivendo precisi limiti al taglio degli alberi, tanto da stabilire che ogni anno se ne sarebbero potuto utilizzare solo 12 unità tra roveri e cerri, mentre per le altre specie non venne previsto alcun limite (Fumagalli, 1976, p. 10). Ancora boschi, sfruttati questa volta per il taglio della legna, materiale di valore da impiegare per molteplici scopi, soprattutto le essenze più pregiate, quelle che opportunamente vennero tutelate (Cortonesi, 2006, pp. 23-24) impedendone il taglio indiscriminato (Andreolli 2000, p. 435). Tra quanto la contessa trasferì a Polirone nel 1115 v'erano uomini addetti allo svolgimento di diverse attività legate al fiume, allo sfruttamento delle terre, delle selve e delle valli, e pure alla navigazione, al trasporto e alla pesca. Alla fine degli anni Venti, il marchese Alberto di San Bonifacio donò all'abate di San Benedetto alcuni mansi siti nella selva di Pegognaga, ubicati nel luogo chiamato Castiniolo, confinanti con altri beni del donatore e del destinatario e a settentrione con il Po vecchio, nonché tutta la terra "que noviter runcata erat iuxta ipsam silvam Castiniolo et iuxta silvam que dicitur Carpaneda", la cui estensione superava di poco i nove iugeri ${ }^{37}$. Detti mansi non dovevano essere stati sino ad allora resi coltivabili se all'abate si riconobbe proprio il diritto di roncarli. Meno di tre mesi dopo, lo stesso marchese investirà il priore del monastero della restante parte della silva de Casteniolo: nel frattempo, sul confine che delimitava questa parte di selva con quella già assegnata all'ente monastico, era stato scavato un fossato; tra i termini di confine, oltre al Po, si citano degli argini, uno dei quali è 'antico's8.

32 Rinaldi et al., 1993, n. 32, 1074 giugno 25.

33 Rinaldi et al., 1993, n. 50, 1096 agosto 13.

34 Rinaldi et al., 1993, n. 83, 1113.

35 Rinaldi et al., 1993, n. 86, 1114 novembre 8.

36 Rinaldi et al., 1993, n. 84, 1113.

37 Rinaldi, Golinelli, 2011, n. 8, 1129 gennaio 25.

38 Rinaldi, Golinelli, 2011, n. 9, 1129 aprile 10. Nel 1134 il marchese Alberto investirà il monastero di San Benedetto di altri beni fondiari posti in Pegognaga: Rinaldi, Golinelli, 2011, n. 26, 1134 febbraio 4. 
Per i nostri scopi sono di particolare utilità alcuni testimoniali prodotti in occasione di controversie giudiziarie aventi per oggetto possedimenti proprio nell'area di nostro interesse. Le ragioni del contendere tra l'ente monastico e diversi soggetti istituzionali - e in particolare l'episcopio mantovano - mettevano a repentaglio la possibilità per gli uomini dell'abbazia - e non solo per loro invero - di cacciare e di allevare liberamente animali, di frequentare boschi e paludi (Rinaldi, 2008, p. 17). Le liti infatti riguardavano per la maggior parte estensioni e fasce confinarie di foreste, valli e corsi d'acqua. In verità, in anni successivi alla morte di Matilde, molti di quei terreni furono convertiti in suolo agricolo: i campi erano ritagliati anche nella striscia di territorio che separava il Po vecchio - in lenta fase di abbandono - dal Po Lirone, destinato come si è detto a divenirne il corso principale, luoghi dove il pericolo di rotte ed esondazioni era costante, luoghi che potevano quindi tornare allo stato 'selvaggio'.

Nell'impossibilità di fornire una minuta disamina di tutta la documentazione, conviene soffermarsi sulle testimonianze rese nel $1189^{39}$, quando l'abate di San Benedetto dovette difendere le proprie prerogative - nel corso di una controversia che si doveva trascinare da decenni - contro le rivendicazioni del vescovo di Mantova ${ }^{40}$ in merito ai diritti di sfruttamento dei terreni e delle acque sull'isola di San Benedetto. I testi ascoltati prodotti da entrambe le parti in causa sono un centinaio e provengono per lo più dagli abitati di Governolo, Nuvolato e Revere. Poco importa a noi a favore di chi essi deponessero, ci interessa richiamare l'attenzione sugli elementi che ci aiutano a comprendere i rapporti tra l'uomo e l'ambiente naturale e come si cercasse di intervenire su di esso per modificarlo. Si narra che il vescovo di Mantova da tempo possedeva un nemus magnum delimitato da fossati - v'erano roveri e ulivastri - controllato da due guardaboschi, uno dipendente da lui e uno dipendente dall'abate di San Benedetto: Ruzmento de Nubilario dice che da quarant'anni "predictum nemus est destructum". È un'affermazione eloquente, che pone in risalto quanto incisiva fosse stata l'azione dell'uomo. A promuoverne il dissodamento erano stati i monaci: "ceperunt runchare in predictis locis". Le paludi da tempo erano meta di diversi pescatori; un fossato (il fossato di San Siro) da trent'anni era stato riempito con gli scarti del taglio degli alberi, ed in particolare dei salici. La sua chiusura arrecò gravi perdite all'episcopio al quale venne in quel modo impedita la possibilità di accedere alle paludi per praticarvi la pesca; quando invece era praticabile lo si percorreva "cum navibus". Altri testi scandiscono nel tempo l'attività di bonifica, iniziata da quarant'anni, ad intervalli vari (30, 20, 10, 5 e 3 anni). Gli uomini del vescovo residenti in Nuvolato erano avvezzi a pascolarvi gli animali, tagliavano legna, cacciavano e pescavano. Lepiscopio mantovano vi aveva propri cacciatori sin dagli anni

39 Rinaldi, Golinelli, 2011, n. 145, 1189 <maggio 30, luglio 5 e 6>; n. 146, 1189 giugno 2, luglio 4 e 27. $40 \mathrm{Il}$ vescovo di Mantova disporrà di diritti di pesca su queste stesse aree ancora al principio del Trecento: ASMn, AG, b. 3174, Fascicolo XIII, anni 1311-1317. 
dell'episcopato di Manfredo; essi cacciavano in "quadam sua propria aia, quam ipse habebat supra dictum fosatum". In quello stesso torno di tempo un polesino era stato reso coltivabile tanto che ora lo si poteva lavorare e arare, come lo stesso teste aveva fatto. Nel corso delle deposizioni si parla spesso di rotte, alla cui riparazione gli uomini delle comunità venivano invitati a collaborare trattandosi di interventi di comune utilità. Gli uomini di Governolo ebbero libera possibilità di pascolare, tagliare legna, tagliare erba, pescare nelle paludi e anche nell'insula di San Benedetto. Sono diversi i fossati nominati (Anguillara, San Siro, Frisia, Lonello) e molte le paludi: Tencacrossam, Salceretum, Cicogneram, Pasenaticum, Brusacos, Marcignagam, Scardevarola). Il teste di nome Sparata dichiarò che gli uomini di San Benedetto inquietarono anche quelli della cattedrale mantovana confermando come i primi da decenni avessero intrapresero opere di messa a coltura che erano ancora in corso.

In quell'area da decenni dunque si operava per sottrarre la terra al bosco e alle acque. I primi attori di quegli interventi erano i membri delle comunità che vi si formarono e consolidarono. Con queste comunità negli anni Novanta del secolo l'abate siglò vari atti d'investitura "ad habitandum": sono i noti 'patti collettivi' (Torelli, 1930, pp. 184-195; Rinaldi, 2005a, pp. 128-129; Rinaldi, 2005b, p. 185) siglati a partire dall'anno 1197 con uomini di Villabona, San Siro e Quistello ${ }^{41}$. I terreni concessi comprendevano porzioni di bosco da roncare e convertire in suolo agricolo: "de terra vero roncanda usque ad tres fruges nichil preter decimam dare debet; dinde rectum quartum et decimam de omnibus in integrum solvat, sicut de roncata ut supra dictum est". L'abitato di Villabona era cresciuto nelle braide abbaziali, ovvero nelle terre agricole di antica acquisizione, solcate da scoli di drenaggio: l'abitato era posto tra il mexonem Padi (area golenale seminata), il nemus de Olmeda e la rotta de Mezopane. Le terre date in concessione si estendono sulle rive del Po Lirone, a sud dell'alveo fluviale, e l'abitato di Governolo e "ubi terra laboratoria poterit inveniri". È evidente: l'abate intese incentivare nuovi interventi di messa a coltura promuovendo nuovi insediamenti, richiamando su quelle terre nuovi abitanti favorendo quindi la mobilità di persone gratificate dalla possibilità di ottenere nuove terra a canoni vantaggiosi. Ad ognuno dei concessionari doveva spettare una unità fondiaria di estensione variabile - analogamente, vien da supporre, al modello dei 'campi a strisce' e ai piani di divisione in mansi noti anche per altre aree (Torelli, 1930, pp. 143-154; Castagnetti, 1974; Camerlenghi, 2010, pp. 85-87) riconoscibili anche nella cartografia storica posteriore (Figura 4) -, una parte della quale doveva essere destinata alla costruzione del casamento. I concessionari erano tenuti alla corresponsione di un canone e di alcune prestazioni d'opera oltre che alla manutenzione degli argini: "aggeres quidem pro defensione suarum terrarum quisque facere tenetur; ad ruptas fondales per totam abaciam pro voluntate nunciorum monasterii venire et laborare

41 Rinaldi, Golinelli, 2011, n. 181, 1197 aprile 24; n. 182, 1197 aprile 24; n. 183, 1197 aprile 24. 
debet". La lotta all'acqua era ancora l'indispensabile garanzia per la buona riuscita di ogni azione di messa a coltura di nuovi terreni. A segnare ancor più la metamorfosi di quei luoghi s'univa la diffusione della vite: "et quisque de uno manso et pro uno plantare debet bubulcam unam ad vineam". Un'altra concessione doveva invogliare ad intraprendere la lotta al bosco (Rinaldi, 2005, p. 118): "de lignis terre quam roncabunt et zapulabunt per II annos nichil dabunt nisi decimam". Sono interventi che proseguiranno nel tempo: l'ente monastico favorisce la mobilità degli uomini, il consolidarsi delle comunità e dei loro abitati, l'agrarizzazione delle sue terre. Nel 1204, ad esempio, una trentina d'uomini vengono investiti dell'isola di Gorgo in modo del tutto simile a quanto era stato pattuito nei contratti del $1197^{42}$. Lanno dopo l'abate Alberto investirà un gruppo di uomini "de tota terra que est posita iuxta ripa Padi veteris, in insula Sancti Benedicti, apud tegetem Sancri Bartolomei, ad habitandum in perpetuum" ovvero di circa tre mansi (i cui confini sono: "a mane, versus Custellum, Padus vetus, a meridie idem Padus, a sero terra monasterii Sancti Bartholomei, a monte nemus"), con l'obbligo di impiantarvi viti' ${ }^{43}$. Nel 1221 in una nuova investitura a favore di una sessantina di persone di terre aratorie e prative di 193 biolche ripartite in 49 lotti di diversa consistenza, si fa diretto riferimento alle pattuizioni del 1197 e i confini dei beni tradiscono il lavoro nel frattempo condotto dagli uomini in quelle terre: "a mane laborationes veteras Sancti Syri, a meridie, via nova, a sera dugalem novum" 4 .

Parrebbe insomma che al principio del Duecento anche le terre prossime al Po avessero oramai mutato il loro aspetto a seguito di un poderoso programma di messa a coltura che implicava necessariamente opere di contenimento delle acque (Andreolli, 2000, pp. 426-431; Gardoni, 2010, pp. 171-175; Mancassola, 2012, pp. 126-127). Non è qui il caso di procedere oltre, magari per mostrare come quel processo fosse in corso ancora molti decenni dopo o per cercare di appurare se quegli interventi di bonifica non siano stati in qualche caso assai precari ed abbiano portato a risultati di breve durata. Di certo non si può mettere in dubbio il fatto che anche quell'area perifluviale avesse subito una metamorfosi al pari di quel che, ad esempio, doveva essere accaduto nelle paludi di Bagnolo e Formigada di proprietà del monastero cittadino di Sant'Andrea che agli inizi del secolo XIII ne aveva intrapreso la bonifica imponendo di piantare ed allevare salici ma riservandosi i diritti di pesca e di caccia ${ }^{45}$. Non molto lontane erano poi quei beni della cattedrale mantovana che in quel medesimo periodo risultavano essere state dissodate da 25 anni ed essere come si è detto all'inizio di questi appunti - "ad usum panis reducte".

42 ASMn, Corporazioni religiose soppresse. San Benedetto, vol. 526, c. 8r, 1204 marzo 3.

43 ASMn, Corporazioni religiose soppresse. San Benedetto, vol. 526, c. 21r, 1205 novembre 8.

44 ASMn, Corporazioni religiose soppresse. San Benedetto, vol. 526, c. 17r, 1221 febbraio 21.

45 Nicolini, 1949, n. LXXIV, 1208 marzo 5; n. LXXVIII, 1209 marzo 22; n. LXXXII, 1210 febbraio 7; n. LXXXIII, 1210 maggio 30. 


\section{REFERENZE BIBLIOGRAFICHE}

ANDREOLLi B. (1998). "De nemore inciso et pasquo arato». I caratteri originali della patrimonialità polironiana, En P. Golinelli (ed.), Storia di San Benedetto Polirone. Le origini (961-1125) (pp. 141-151). Bologna: Pàtron.

ANDREOLLI B. (1999). Contadini su terre di signori. Studi sulla contrattualistica agraria dell'Italia medievale. Bologna: Clueb.

Andreolli B. (2000). Il Po tra alto e basso Medioevo: una civiltà idraulica, En C. Ferrari, L. Gambi (Ed.). Un Po di terra. Guida all'ambiente della bassa pianura padana e alla sua storia (pp. 415-443). Reggio Emilia: Diabasis.

ANDREOLli B. (2015). Selve, boschi, foreste tra alto e basso medioevo. En I paesaggi agrari d'Europa (secoli XIII-XV) (pp. 385-431). Roma: Viella.

Andreolli B., Fumagalli V., Montanari M. (Ed.). (1985). Le campagne italiane prima e dopo il Mille. Una società in trasformazione. Bologna: Clueb.

Andreolli B., Galetti P., Lazzari T., Montanari M. (Ed.). (2010). Il medioevo di Vito Fumagalli. Spoleto: CISAM.

ANDREOlli B., MONTANARI M. (1985). L'azienda curtense in Italia. Proprietà della terra e lavoro contadino nei secoli VIII-XI. Bologna: Clueb.

Andreolli B., Montanari M. (Ed.). (1988). Il bosco nel medioevo. Bologna: Clueb.

ARChetTi G. (1998), Tempus vindemie. Per la storia delle vigne e del vino nell'Europa medievale. Brescia: Civiltà bresciana.

ArChetti G. (Ed.). (2019). Le carte della «curtis» di Migliarina, 767-1200. Roma: Studium.

BonACini P.P. (2000). Il monastero di San Benedetto Polirone: formazione del patrimonio fondiario e rapporti con l'aristocrazia italica nei secoli XI e XII, Archivio storico italiano, CLVIII, 623-678.

BONACINI P.P. (2001). Terre d'Emilia. Distretti pubblici, comunità locali e poteri signorili nell'esperienza di una regione italiana (secoli VIII-XII). Bologna: Clueb.

BRANDOLINI F. (2018). The Impact of Late Holocene Flood Management on the Central Po Plain (Northen Italy), Sustainability, 10, https://doi.org/10.3390/sul0113968

Brandolini F., CRemaschi M. (2018). Valli-Paludi nel Medioevo: il rapporto tra uomo e acque nella Bassa Pianura Reggiana. Le bonifiche "laiche" per colmata. En Sogliani F., Gargiulo B., Annunziata E., Vitale V. (Eds), VIII Congresso Nazionale di Archeologia Medievale, 2, Territorio e Paesaggio (pp. 72-78). Sesto Fiorentino (FI): All'Insegna del Giglio.

BRANDOLini F (2019). Estimating the Potential of Archaeo-historical Data in the Definition of Geomorphosites and Geo-educational Itineraries in the Central Po Plain (N Italy), Geoheritage, (11), 1371-1396. https://doi.org/10.1007/s12371-019-00370-5

BRANDOLINI F. (2020). Late-Holocene human resilience in a fluvial environment: a geoarchaeological database for the Central Po Plain (N Italy). The Journal of Open Archaelogy Data, (8). https://doi.org/10.5334/joad.62. 
Brandolini F., Carrer F. (2020). Terra, Silva et Paludes. Assessing the Role of Alluvial Geomorpghology for Late-Holocene Settlement Strategies (Po Plain-N. Italy) Through Point Pattern Analys, Environmental Archaelogy. The Journal of Human Palaeoecology, 1-15. https://doi.org/10.1080/14614103.2020.1740866

CaVAlazZi M., Mancassola N. (2021). Linsediamento rurale nel territorio ravennate tra la tarda Antichità e l'alto Medioevo (IV-XI secolo), En Galetti P. (Ed.), Una terra antica. Paesaggi e insediamenti nella Romania dei secoli V-XI (pp. 63-72). Sesto Fiorentino (FI): All'Insegna del Giglio.

Bresslau H. (Ed.). (1909). Diplomata regum et imperatorum Germaniae, IV, Die Urkunden Konrads II. Hannover: Monumenta Germaniae Historica.

BRÜHL C. (Ed.). (1973). Codice diplomatico longobardo, III. Roma: Istituto storico italiano per il Medioevo.

BuOnOPANE A. (2003). Il popolamento nell'ager di Mantua: la documentazione epigrafica, En Il paesaggio mantovano nelle tracce materiali, nelle lettere e nelle arti, I, Dalla preistoria all'età tardo romana (pp. 111- 124). Firenze: Olschki.

CAlzOlari M. (1989). Padania romana. Ricerche archeologiche e paleoambientali nella pianura tra il Mincio e il Tartaro. Mantova: Tipo-Litografia Dini.

Calzolari M. (1998). Il territorio di San Benedetto di Polirone: idrografia e topografia nell'alto medioevo. En P. Golinelli (ed), Storia di San Benedetto Polirone. Le origini (961-1125) (pp. 1-33). Bologna: Pàtron.

Calzolari M. (2003). Divisioni agrarie di età romana nel territorio mantovano: problemi e ipotesi, En Il paesaggio mantovano nelle tracce materiali, nelle lettere e nelle arti, I, Dalla preistoria all'età tardo romana (pp. 139-161). Firenze: Olschki.

CAMERlenghi E. (2005). Agricoltura e paesaggio agrario medievale nell'opera di Pietro Torelli. En Il paesaggio mantovano nelle tracce materiali, nelle lettere e nelle arti, II, Il paesaggio mantovano nel Medioevo (pp. 133-153). Firenze: Olschki.

CAmerlenghi E. (2010). Il monastero e l'economia rurale, En Ambrosini C., De Marchi P.M. (Ed). Uomini e acque a San Benedetto Po. Il governo del territorio tra passato e futuro (pp. 85-89). Borgo San Lorenzo (FI): All'Insegna del Giglio.

CAstagnetti A. (1969). La distribuzione geografica dei possessi di un grande proprietario veronese del secolo IX: Enghelberto del fu Grimoaldo di Erbè. Rivista di storia dell'agricoltura, (IX/1), 15-26.

Castagnetti A. (1972). I possessi del monastero di San Zeno di Verona a Bardolino. Studi medievali, XIII, 95-159.

Castagnetti A. (1974). Primi aspetti di politica annonaria nell'Italia comunale. La bonifica della 'palus comunis Verone' (1194-1999). Studi medievali, (15), 363481.

Castagnetti A. (1974-1975). Contributo allo studio dei rapporti fra città e contado. Le vicende del castello di Villimpenta dal X al XIII secolo. En Atti dell'Istituto veneto di scienze, lettere ed arti, CXXXIII, 81-137. 
CASTAGNETTI A. (1977). La pianura veronese nel medioevo. La conquista del suolo e la regolamentazione delle acque. En G. Borrelli (Ed). Una città e il suo fiume. Verona e l'Adige (pp. 35-138). Verona: Banca Popolare di Verona.

Castagnetti A., Luzzati M., Pasquali G., Vasina A. (Ed.) (1979). Inventari altomedievali di terre, coloni e redditi. Roma: Istituto storico italiano per il Medioevo.

Castagnetti A. (1990). La "campanea" e i beni comuni delle città. En L'ambiente vegetale nell'alto medioevo (pp. 137-174). Spoleto: CISAM.

Castagnetti A. (1996). Arimanni in 'Langobardia' e in 'Romania' dall'età carolingia all'età comunale. Verona: Libreria universitaria editrice.

Castagnetti A., Ciaralli A. (2011). Falsari a Nonantola. I placiti di Ostiglia (820827) e le donazioni di Nogara (910-911). Spoleto: CISAM.

Cavalazzi M. (2019). Un ordine fragile. La costruzione del distretto del Comune di Reggio Emilia (XII-XIII secolo). Bologna: Bononia University Press.

COLORNI V. (1959). Il territorio mantovano nel sacro romano impero, I, Periodo comitale e periodo comunale (800-1274). Milano: Giuffré.

Comba R. (1983). Metamorfosi di un paesaggio rurale. Uomini e luoghi del Piemonte sud-occidentale dal X al XVI secolo. Torino: Celid.

CORTONESI A. (1995). Ruralia. Economie e paesaggi del medioevo italiano. Roma: il Calamo.

CORTONESi A., MONTANARI M. (Ed.) (2001). Medievistica italiana e storia agraria. Bologna: Clueb.

CORTONESI A. (2006). L'agricoltura italiana fra XIII e XIV secolo: vecchi e nuovi paesaggi. En Cortonesi A., Piccinni G. (ed), Medioevo delle campagne (pp. 15-55). Roma: Viella.

DeVroey J.P., Montanari M. (2009), Città, campagna, sistema curtense (secoli IX-X), in Città e campagna nei secoli altomedievali (pp. 777-808). Spoleto: Cisam.

FRANCESCHINI A. (1991). Giurisdizione episcopale e comunità rurali altopolesane. Bergantino Melara Bariano Trecenta [sec. X-XIV]. Documenti. Bologna: Pàtron.

FuMAGAlli V. (1976). Terra e società nell'Italia padana. I secoli IX e X, Torino: Einaudi.

Fumagalli V. (1985). Città e campagna nell'Italia medievale. Bologna: Pàtron.

Fumagalli V. (1986). Il Regno italico. Torino: Utet.

Fumagalli V. (1989). Uomini e paesaggi medievali. Bologna: Il Mulino.

Fumagalli V. (1992). L’uomo e l'ambiente nel medioevo. Roma-Bari: Laterza.

Galetti P. (2019). Paesaggi agrari della bassa pianura emiliana tra XI-XIII secolo: il caso del territorio reggiano, Edad Media. Revista de Historia, (20), 113-156. https://doi.org/10.24197/em.20.2019.113-156

GARDONI G. (2006). Vescovi e città a Mantova dall'età carolingia al secolo XI. En Le origini della diocesi di Mantova e le sedi episcopali dell'Italia settentrionale (IV-XI secolo) (pp.183-246). Trieste: Editreg. 
GARDONI G. (2007). Élites cittadine fra XI e XII secolo: il caso mantovano. En A. Castagnetti, A. Ciaralli, G.M. Varanini (Ed.). Medioevo. Studi e documenti, II (pp. 279-348). Verona: Libreria universitaria editrice.

GARDONI G. (2010). Uomini e acque nel territorio mantovano (secoli XI-XIII). En Calzona A., Lamberini D. (Ed.), La civiltà delle acque tra Medioevo e Rinascimento (pp. 143-176). Firenze: Olschki.

Gaulin J.L., GRIECO A. (Ed.). (1994). Dalla vite al vino. Fonti e problemi della vitivinicoltura italiana medievale. Bologna: Clueb.

Goez E., Goez W. (Ed). (1998). Die Urkunden und Briefe der Markgräfin Mathilde von Tuszien. Hannover: Hahnsche Buchhandlung.

Golinelli P. (Ed). (1998). Storia di San Benedetto Polirone. Le origini (961-1125). Bologna: Pàtron.

Golinelli P. (Ed.). (2008). L'abbazia di Matilde. Arte e storia in un grande monastero dell'Europa benedettina. Bologna: Pàtron.

MANARESI C. (Ed.). (1955). I placiti del Regnum Italiae. Roma: Istituto storico italiano per il Medioevo.

MANCASSOla N. (2008). L'azienda curtense tra Longobardia e Romania. Rapporto di lavoro e patti colonici dall'età carolingia al Mille. Bologna: Clueb.

MANCASSOLA N. (2012). Uomini e acque nella pianura reggiana durante il Medioevo (secoli IX-XIV). En D. Canzian, R. Simonetti (Ed.). Acque e territorio nel Veneto medievale (pp. 115-129). Roma: Viella.

Mancassola N. (2018). Il patrimonio fondiario del monastero di San Silvestro di Nonantola in età carolingia: insediamenti e comunità nella bassa pianura lungo il corso del Po. En P. Golinelli, G. Malaguti (Ed.). Nonantola e il territorio modenese in età carolingia (pp. 87-103). Bologna: Pàtron.

MancAssola N. (2019). La corte di Migliarina. Signori, rustici e comunità nella valle del Po tra VIII e XII secolo. En G. Archetti (Ed.). Le carte della «curtis» di Migliarina, 767-1200 (pp. 11-47). Roma: Studium.

Marchetti M. (2002). Environmental changes in the Central Po Plain (northern Italy) due to fluvial modifications and anthropogenic activities, Geomorphology, (44), 361-373. https://doi.org/10.1016/S0169-555X(01)00183-0

Marchetti M. (2010). Persistenze di morfologie originali e loro relazione con la struttura antropica. En Ambrosini C., De Marchi P.M. (Ed). Uomini e acque a San Benedetto Po. Il governo del territorio tra passato e futuro (pp. 25-30). Borgo San Lorenzo (FI): All'Insegna del Giglio.

Menant F. (1993). Campagnes lombardes du Moyen Âge. L'économie et la société rurales dans la région de Bergame, de Crémone et de Brescia du X $X^{e}$ au XIII ${ }^{e}$ siécle. Rome: Ècole française de Rome. https://doi.org/10.3406/befar.1993.1232

MONTANARI M. (1984). Campagne medievali. Strutture produttive, rapporti di lavoro, sistemi alimentari. Torino: Einaudi. 
Mor C.G. (1964). Dalla caduta dell'Impero al Comune. En, Verona e il suo territorio, II, Verona medioevale. (pp. 5-242). Verona: Istituto per gli studi storici veronesi. NAVARRINi R. (Ed.) (1988). Liber privilegiorum comunis Mantue. Mantova: Arcari.

Nicolini U. (Ed.) (1949). Larchivio del monastero di Sant'Andrea di Mantova. Mantova: Accademia Virgiliana.

PAGLIA E. (1879). Saggio di studi naturali sul territorio mantovano. Mantova: Guastalla. Parmigiani C. (2016). Dal Padus al Po. Origini, evoluzione e cartografia del Po mantovano. Mantova: Sometti.

PARMigIANi C. (2019). Mantova e l'acqua amore antico. Storia per immagini del governo delle acque in città e nel contado, dal Medioevo alle soglie del Novecento. Mantova: Sometti.

PASQUAli G. (2005). Il lavoro contadino e la produzione agricola: tradizione e innovazione sulle terre mantovane (secoli VIII-X). En Il paesaggio mantovano nelle tracce materiali, nelle lettere e nelle arti, II, Il paesaggio mantovano nel Medioevo (pp. 155-168). Firenze: Olschki.

PASQUAli G. (2008). Sistemi di produzione agraria e aziende curtensi nell'Italia altomedievale. Bologna: Clueb.

Pini A.I. (1989). Vite e vino nel medioevo. Bologna: Clueb.

RAO R. (2015). I paesaggi dell'Italia medievale. Roma: Carocci.

RAO R. (2016). Metamorfosi di un paesaggio fluviale: uomini e luoghi sulla sponda sinistra del Po vercellese dal X al XV secolo. En Medioevo vissuto. Studi per Rinaldo Comba fra Piemonte e Lombardia (pp. 63-82). Roma: Viella.

Rinaldi R. (1998). Un'abbazia di famiglia. La fondazione di Polirone e i Canossa. En P. Golinelli (ed.), Storia di San Benedetto Polirone. Le origini (961-1125) (pp. 3554). Bologna: Pàtron.

RiNALDI R. (2005a). Il fiume mobile. Il Po mantovano tra monaci-signori, vescovi cittadini e comunità (secoli XI-XII), En Il paesaggio mantovano nelle tracce materiali, nelle lettere e nelle arti, II, Il paesaggio mantovano nel Medioevo (pp. 113-131). Firenze: Olschki.

Rinaldi R. (2005b). Dalla via Emilia al Po. Il disegno del territorio e i segni del popolamento (secc. VIII-XIV). Bologna: Clueb.

RinAldi R. (2008). Monaci, contadini e ambiente (secc. X-XII). En P. Golinelli (Ed.). L'abbazia di Matilde. Arte e storia in un grande monastero dell'Europa benedettina (pp. 13-19). Bologna: Pàtron.

Rinaldi R., Villani C., Golinelli P. (Ed.). (1993). Codice diplomatico polironiano (961-1125). Bologna: Pàtron.

Rinaldi R., Golinelli P. (Ed.). (2011). Codice diplomatico polironiano, II (11261200). Bologna: Pàtron.

SAggioro F. (2010). Paesaggi di pianura: trasformazioni del popolamento tra Età romana e Medioevo. Borgo S. Lorenzo (FI): All'Insegna del Giglio. 
SChiaparelli L. (Ed.) (1903). I diplomi di Berengario I, Roma: Forzani.

SERENI E. (1963). Storia del paesaggio agrario italiano. Bari: Laterza.

SiCKel T. (Ed.). (1893). Diplomata regum et imperatorum Germaniae, II/2. Die Urkunden Otto des III. Hannover: Monumenta Germaniae Historica.

Tamassia A.M. (Ed.) (1996). Archeologia di un ambiente padano. S. Lorenzo di Pegognaga (Mantova). Firenze: Olschki.

TAMASSIA A.M. (2003). Stanziamenti e frequentazione nel territorio mantovano in età romana, En Il paesaggio mantovano nelle tracce materiali, nelle lettere e nelle arti, I, Dalla preistoria all'età tardo romana (pp. 163-182). Firenze: Olschki.

TORELLI P. (1930). Un comune cittadino in territorio ad economia agricola, I, Distribuzione della proprietà, sviluppo agricolo, contratti agrari. Mantova: Accademia Virgiliana.

TORelli P. (Ed.). (1914). Regesto mantovano. Le carte degli archivi Gonzaga e di Stato in Mantova e dei monasteri Mantovani soppressi (Archivio di Stato di Milano). Roma: Ermanno Loescher.

TORELli P. (Ed.). (1924). L'archivio capitolare della cattedrale di Mantova fino alla caduta dei Bonacolsi. Verona: Mondadori.

VARANINI G.M., SAGGIORO F. (2008). Ricerche sul paesaggio e sull'insediamento d'età medievale in area veronese. En S. Lusuardi Sena (Ed.). Dalla curtis alla pieve fra archeologia e storia. Territori a confronto: l'oltrepo' pavese e la pianura veronese (pp. 101-160). Mantova: SAP.

VARANINI G. M., SAGGIORO F. (2010). Insediamento umano, terra e acque nella pianura veronese (IX-XIV secolo): archeologia e fonti scritte. En D. Canzian, R. Simonetti (Ed.). Acque e territorio nel Veneto medievale (pp. 95-114). Roma: Viella. 